\title{
Global Hopf Bifurcation on Two-Delays Leslie-Gower Predator-Prey System with a Prey Refuge
}

\author{
Qingsong Liu, ${ }^{1}$ Yiping Lin, ${ }^{1}$ and Jingnan $\mathrm{Cao}^{2}$ \\ ${ }^{1}$ Department of Applied Mathematics, Kunming University of Science and Technology, Kunming, Yunnan 650500, China \\ ${ }^{2}$ School of Computer Science, Beijing University of Post Telecommunication, Beijing 100876, China \\ Correspondence should be addressed to Yiping Lin; linyiping689@163.com
}

Received 16 December 2013; Accepted 18 January 2014; Published 7 April 2014

Academic Editor: Chengjun Sun

Copyright (c) 2014 Qingsong Liu et al. This is an open access article distributed under the Creative Commons Attribution License, which permits unrestricted use, distribution, and reproduction in any medium, provided the original work is properly cited.

\begin{abstract}
A modified Leslie-Gower predator-prey system with two delays is investigated. By choosing $\tau_{1}$ and $\tau_{2}$ as bifurcation parameters, we show that the Hopf bifurcations occur when time delay crosses some critical values. Moreover, we derive the equation describing the flow on the center manifold; then we give the formula for determining the direction of the Hopf bifurcation and the stability of bifurcating periodic solutions. Numerical simulations are carried out to illustrate the theoretical results and chaotic behaviors are observed. Finally, using a global Hopf bifurcation theorem for functional differential equations, we show the global existence of the periodic solutions.
\end{abstract}

\section{Introduction}

The dynamic relationship between predators and their prey has long been and will continue to be one of the dominant topics, not only in ecology but also in mathematical ecology due to its universal existence and importance. In [1], Leslie introduced a predator-prey model in which the "carrying capacity" of the predator's environment is proportional to the number of prey:

$$
\begin{aligned}
& \dot{x}=r_{1} x\left(1-\frac{x}{K}\right)-a x y, \\
& \dot{y}=r_{2} y\left(1-\frac{y}{\gamma x}\right),
\end{aligned}
$$

where $r_{1}, r_{2}, K$, and $\gamma$ are positive constants and $x(t)$ and $y(t)$ denote the population of the prey and predator at time $t$, respectively. The parameters $r_{1}$ and $r_{2}$ are the intrinsic growth rates of the prey and the predator. The value $K$ is the carrying capacity of the prey, and $\gamma x$ takes on the role of a prey-dependent carrying capacity for the predator; the parameter $\gamma$ is a measure of the quality of the prey as food for the predator. However, this model has attracted the attention of some authors [2-4].
Time delays are often incorporated into population models for resource regeneration times, for example, maturing times and gestation periods $[5,6]$. Recently, great attention has been received and a lot of work has been carried out on the existence of the Hopf bifurcations in delayed population models (see [7-9] and references cited therein). The stability of positive equilibria and the existence and the direction of the Hopf bifurcations were discussed, respectively, in the references mentioned above. In [10], Yuan and Song considered the following delayed Leslie-Gower predator-prey system:

$$
\begin{aligned}
& \dot{x}(t)=r_{1} x(t)\left(1-\frac{x(t-\tau)}{K}\right)-a x(t) y(t), \\
& \dot{y}(t)=r_{2} y(t)\left(1-\frac{y(t)}{\gamma x(t)}\right) .
\end{aligned}
$$

They investigated the stability and the Hopf bifurcation of the above system without considering the effects of time delay on predator.

Motivated by the above discussion, in this paper, by choosing the time delays $\tau_{1}$ and $\tau_{2}$ as bifurcation parameters, 
we investigate a modified Leslie-Gower predator-prey system with two delays described by the following system:

$$
\begin{aligned}
& \dot{x}(t)=r_{1} x(t)\left(1-\frac{x\left(t-\tau_{1}\right)}{K}\right)-a(1-m) x(t) y(t), \\
& \dot{y}(t)=r_{2} y(t)\left(1-\frac{y\left(t-\tau_{2}\right)}{\gamma(1-m) x\left(t-\tau_{2}\right)}\right),
\end{aligned}
$$

where $\tau_{1}$ and $\tau_{2}$ are all positive constants. Due to crowding, the prey dynamics is delayed by $\tau_{1}$ [11]. The negative feedback delay $\tau_{2}$ is assumed in predator growth [12]. $\mathrm{mH}$ is a refuge protecting of the prey and $m \in[0,1)$ is a constant. This leaves $(1-m) H$ of the prey available to the predator.

The initial conditions for system (3) take the from

$$
\begin{gathered}
x(\theta)=\varphi(\theta), \quad y(\theta)=\psi(\theta), \\
\varphi(\theta)>0, \quad \psi(\theta)>0, \quad \theta \in[-\tau, 0],
\end{gathered}
$$

where $(\varphi(\theta), \psi(\theta)) \in\left([-\tau, 0], R_{+0}^{2}\right), R_{+0}^{2}=\left\{\left(x_{1}, x_{2}\right): x_{i}>\right.$ $0, i=1,2\}$.

This paper is organized as follows. In Section 2, we investigate the effect of two delays $\tau_{1}$ and $\tau_{2}$ on the stability of the positive equilibrium of system (3). In Section 3, we derive the direction and stability of the Hopf bifurcation by using normal form and central manifold theory. In Section 4, numerical simulations are performed to support the stability results and chaos is observed. Finally, in Section 5, based on the global Hopf bifurcation theorem for general functional differential equations, we investigate the global existence of periodic solutions by using degree theory methods.

\section{Local Stability Analysis and the Hopf Bifurcation}

It is easy to see that system (3) has a unique positive equilibrium $E_{*}\left(x_{*}, y_{*}\right)$, where

$$
x_{*}=\frac{K r_{1}}{r_{1}+a K \gamma(1-m)^{2}}, \quad y_{*}=\gamma(1-m) x_{*} .
$$

Let $\bar{x}=x-x_{*}, \bar{y}=y-y_{*}$ and still denote by $\bar{x}=x$, $\bar{y}=x$; system (3) can be written as

$$
\begin{aligned}
\dot{x}= & \alpha_{1} y(t)+\alpha_{2} x\left(t-\tau_{1}\right)+\alpha_{3} x(t) x\left(t-\tau_{1}\right)+\alpha_{4} x(t) y(t), \\
\dot{y}= & \alpha_{5} x\left(t-\tau_{2}\right)+\alpha_{6} y\left(t-\tau_{2}\right) \\
& +\sum_{i+j+k \geq 2} \frac{1}{i ! j ! k !} f_{i j k} x^{i}\left(t-\tau_{2}\right) y^{j}\left(t-\tau_{2}\right) y^{k}(t),
\end{aligned}
$$

where

$$
\begin{gathered}
\alpha_{1}=-a(1-m) x_{*}, \quad \alpha_{2}=-\frac{r_{1} x_{*}}{K}, \quad \alpha_{3}=-\frac{r_{1}}{K}, \\
\alpha_{4}=-a(1-m), \quad \alpha_{5}=\gamma(1-m) r_{2}, \quad \alpha_{6}=-r_{2}, \\
f=r_{2} y_{1}\left(1-\frac{y}{\gamma(1-m) x}\right), \quad f_{i j k}=\left.\frac{\partial^{i+j+k} f^{(1)}}{\partial x^{i} \partial y^{j} \partial k^{k}}\right|_{\left(x_{*}, y_{*}, y_{*}\right)} .
\end{gathered}
$$

We then obtain the linearized system

$$
\begin{aligned}
& \dot{x}=\alpha_{1} y(t)+\alpha_{2} x\left(t-\tau_{1}\right), \\
& \dot{y}=\alpha_{5} x\left(t-\tau_{2}\right)+\alpha_{6} y\left(t-\tau_{2}\right) .
\end{aligned}
$$

The corresponding characteristic equation is

$$
\lambda^{2}-(A \lambda+B) e^{-\lambda \tau_{2}}-C \lambda e^{-\lambda \tau_{1}}+E e^{-\lambda\left(\tau_{1}+\tau_{2}\right)}=0
$$

where

$$
A=\alpha_{6}, \quad B=\alpha_{1} \alpha_{5}, \quad C=\alpha_{2}, \quad E=\alpha_{2} \alpha_{6} .
$$

Case 1. For $\tau_{1}=\tau_{2}=0$, (9) becomes

$$
\lambda^{2}-(A+C) \lambda-B+E=0 .
$$

Since $A+C<0,-B+E>0$, we know that all roots have negative real parts.

Theorem 1. For $\tau_{1}=\tau_{2}=0$, the interior equilibrium point $\left(x_{*}, y_{*}\right)$ is locally asymptotically stable.

Case 2. Consider

$$
\tau_{1}=0, \quad \tau_{2}>0
$$

Theorem 2. For $\tau_{1}=0$, the interior equilibrium point $E_{*}=$ $\left(x_{*}, y_{*}\right)$ is locally asymptotically stable for $0<\tau_{2}<\tau_{2_{0}}$ and it undergoes the Hopf bifurcation at $\tau_{2}=\tau_{2_{0}}$ given by

$$
\tau_{2_{0}}=\frac{1}{\omega_{2_{0}}} \arccos \left[\frac{(E-B-A C) \omega_{1_{0}}^{2}}{A^{2} \omega_{2_{0}}^{2}+(B-E)^{2}}\right] .
$$

Proof. On substituting $\tau_{1}=0$, the characteristic equation (9) becomes

$$
\lambda^{2}-C \lambda-(A \lambda+B-E) e^{-\lambda \tau_{2}}=0 .
$$

Let $i \omega(\omega>0)$ be a purely imaginary root of (14); then it follows that

$$
\begin{aligned}
& A \omega \sin \omega \tau_{2}+(B-E) \cos \omega \tau_{2}=-\omega^{2}, \\
& A \omega \cos \omega \tau_{2}-(B-E) \sin \omega \tau_{2}=-C \omega .
\end{aligned}
$$

Squaring both sides and adding them up, we get the following polynomial equation:

$$
\omega^{4}+\left(C^{2}-A^{2}\right) \omega^{2}-(B-E)^{2}=0
$$

It is easy to know that (16) has unique positive root $\omega_{2_{0}}^{2}$; then the corresponding critical value of time delay $\tau_{2_{n}}$ is

$$
\tau_{2_{n}}=\frac{1}{\omega_{2_{0}}} \arccos \left[\frac{(E-B-A C) \omega_{2_{0}}^{2}}{A^{2} \omega_{2_{0}}^{2}+(B-E)^{2}}\right]+\frac{2 n \pi}{\omega_{2_{0}}},
$$

$$
n=0,1,2, \ldots
$$


Let $\lambda\left(\tau_{2_{n}}\right)= \pm i \omega_{2_{0}}$ be the root of (14); then the transversal condition can be obtained:

$$
\left(\frac{d \lambda}{d \tau_{2}}\right)_{\tau_{2}=\tau_{2_{n}}}^{-1}=\frac{(C-2 \lambda) e^{\lambda \tau_{2}}}{\lambda(A \lambda+B-E)}+\frac{A}{\lambda(A \lambda+B-E)}-\frac{\tau_{2}}{\lambda} .
$$

Since

$$
\begin{aligned}
\operatorname{Sign}\left\{\frac{d\left(\operatorname{Re} \lambda\left(\tau_{2}\right)\right)}{d \tau_{2}}\right\}_{\tau_{2}=\tau_{2 n}}^{-1} & \operatorname{Sign}\left\{\left[\operatorname{Re} \frac{(C-2 \lambda) e^{\lambda \tau_{2}}}{\lambda(A \lambda+B-E)}\right]_{\tau_{2}=\tau_{2 n}}\right. \\
& \left.+\left[\operatorname{Re} \frac{A}{\lambda(A \lambda+B-E)}\right]_{\tau_{2}=\tau_{2 n}}\right\},
\end{aligned}
$$

we can obtain

$$
\begin{gathered}
\operatorname{Sign}\left\{\frac{d\left(\operatorname{Re} \lambda\left(\tau_{2}\right)\right)}{d \tau_{2}}\right\}_{\tau_{2}=\tau_{2 n}}^{-1} \\
=\operatorname{Sign}\left\{\operatorname{Re}\left[\frac{C \cos \omega_{2_{0}} \tau_{2}+2 \omega_{2_{0}} \sin \omega_{2_{0}} \tau_{2}}{-A \omega_{2_{0}}^{2}+i(B-E) \omega_{2_{0}}}\right]\right. \\
\left.+\operatorname{Re}\left[i \frac{C \sin \omega_{2_{0}} \tau_{2}-2 \omega_{2_{0}} \cos \omega_{2_{0}} \tau_{2}}{-A \omega_{2_{0}}^{2}+i(B-E) \omega_{2_{0}}}\right]\right\} \\
\left.+\operatorname{Re}\left[\frac{C}{-A \omega_{2_{0}}^{2}+i(B-E) \omega_{2_{0}}}\right]\right\} \\
=\operatorname{Sign}\left\{\frac{\left(C^{2}-A^{2}\right)+2 \omega_{2_{0}}^{2}}{A^{2} \omega_{2_{0}}^{4}+(B-E)^{2} \omega_{2_{0}}^{2}}\right\} \\
=\operatorname{Sign} \frac{\sqrt{\left(C^{2}-A^{2}\right)^{2}+4(B-E)^{2}}}{A^{2} \omega_{2_{0}}^{2}+(B-E)^{2}}>0,
\end{gathered}
$$

and then we can obtain

$$
\left.\frac{d(\operatorname{Re} \lambda)}{d \tau_{2}}\right|_{\tau_{2}=\tau_{2_{0}}}>0
$$

Case 3. Consider

$$
\tau_{2}=0, \quad \tau_{1}>0
$$

Theorem 3. If $\tau_{2}=0$ holds, the interior equilibrium point $E_{*}\left(x_{*}, y_{*}\right)$ is locally asymptotically stable for $0<\tau_{1}<\tau_{1_{0}}$ and it undergoes the Hopf bifurcation at $\tau_{1}=\tau_{1_{0}}$ given by

$$
\tau_{1_{0}}=\frac{1}{\omega_{1_{0}}} \arccos \left(\frac{\left(-\omega_{1_{0}}^{2}+B\right) E-A C \omega_{1_{0}}^{2}}{E^{2}+C^{2} \omega_{1_{0}}^{2}}\right),
$$

Proof. The proof is similar to that in Case 2.

Case 4. $\tau_{2}$ is fixed in the interval $\left(0, \tau_{20}\right)$ and $\tau_{1}>0$.

Theorem 4. Assume that $B+E>0$ and $\tau_{2} \in\left(0, \tau_{2_{0}}\right)$; then the equilibrium $E_{*}\left(x_{*}, y_{*}\right)$ is asymptotically stable for $\tau_{1} \in\left(0, \tau_{1_{0}}^{\prime}\right)$; moreover let $\left(H_{1}\right)$ hold; $\left(H_{1}\right)$ is defined below; then system (3) undergoes the Hopf bifurcation at $E_{*}\left(x_{*}, y_{*}\right)$ when $\tau_{1}=\tau_{1_{0}}^{\prime}$, where

$$
\tau_{1_{0}}^{\prime}=\frac{1}{\omega_{*}} \arccos \left[\frac{E_{2}\left(k_{2}-k_{3}\right)-E_{1}\left(k_{1}-k_{2}-k_{3}\right)}{E_{1}^{2}+E_{2}^{2}}\right] .
$$

Proof. We know $\tau_{2}$ in its stable interval and $\tau_{1}$ is considered as a parameter. Let $i \omega(\omega>0)$ be a root of (9). Separating real and imaginary parts, leads to

$$
\begin{aligned}
-\omega^{2} & -B \cos \omega \tau_{2}-(A+C) \omega \sin \omega \tau_{2} \\
& =-E \cos \omega \tau_{2} \cos \omega \tau_{1}+E \sin \omega \tau_{2} \sin \omega \tau_{1}, \\
B \sin \omega \tau_{2}-(A+C) \omega \cos \omega \tau_{2} & \\
= & E \cos \omega \tau_{2} \sin \omega \tau_{1}+E \sin \omega \tau_{2} \cos \omega \tau_{1} .
\end{aligned}
$$

Let

$$
H(\omega)=\omega^{4}+l_{1} \omega^{3}+l_{2} \omega^{2}+l_{3}=0
$$

where

$$
\begin{aligned}
& l_{1}=2(A+C) \sin \omega \tau_{2}, \\
& l_{2}=B+A+2 A C+C^{2}, \\
& l_{3}=B^{2}-E^{2} .
\end{aligned}
$$

We assumed that

$$
B+E>0 \text {. }
$$

Then $H(0)<0$ and $H(\infty)=\infty$.

With going detailed analysis (26) it is assumed that there exists at least one real positive root $\omega_{*}$. Now (25) can be written as

$$
\begin{gathered}
k_{1}-k_{2}-k_{3}=-E_{1} \cos \omega_{*} \tau_{1}+E_{2} \sin \omega_{*} \tau_{1}, \\
k_{4}-k_{5}=E_{1} \sin \omega_{*} \tau_{1}+E_{2} \cos \omega_{*} \tau_{1},
\end{gathered}
$$

where

$$
\begin{gathered}
k_{1}=-\omega_{*}^{2}, \quad k_{2}=B \cos \omega_{*} \tau_{2}, \\
k_{3}=(A+C) \omega_{*} \sin \omega_{*} \tau_{2}, \quad k_{4}=B \sin \omega_{*} \tau_{2}, \\
k_{5}=(A+C) \omega_{*} \cos \omega_{*} \tau_{2}, \quad E_{1}=E \cos \omega_{*} \tau_{2}, \\
E_{2}=E \sin \omega_{*} \tau_{2} .
\end{gathered}
$$

Equation (29) is simplified to give

$$
\begin{array}{r}
\tau_{1_{j}}^{\prime}=\frac{1}{\omega_{*}} \arccos \left[\frac{E_{2}\left(k_{4}-k_{5}\right)-E_{1}\left(k_{1}-k_{2}-k_{3}\right)}{E_{1}^{2}+E_{2}^{2}}\right]+\frac{2 j \pi}{\omega_{*}}, \\
j=0,1,2, \ldots,
\end{array}
$$


and $\pm i \omega_{*}$ are purely imaginary roots of $(9)$ for $\tau_{2} \in\left(0, \tau_{2_{0}}\right]$. Now verify the transversal condition of the Hopf bifurcation; differentiating equation (9) with respect to $\tau_{1}$, it is obtained that

$$
\begin{aligned}
\left(\frac{d \lambda}{d \tau_{1}}\right)_{\tau_{1}=\tau_{1 j}^{\prime}}^{-1}= & \left(2 \lambda-A e^{-\lambda \tau_{2}}+(A \lambda+B) \tau_{2} e^{-\lambda \tau_{2}}-C e^{-\lambda \tau_{1 j}^{\prime}}\right. \\
& \left.+C \lambda \tau_{1 j}^{\prime} e^{-\lambda \tau_{1 j}^{\prime}}-\left(\tau_{1 j}^{\prime}+\tau_{2}\right) E e^{-\lambda\left(\tau_{1 j}^{\prime}+\tau_{2}\right)}\right) \\
& \times\left(E \lambda e^{-\lambda\left(\tau_{1 j}^{\prime}+\tau_{2}\right)}-C \lambda^{2} \tau_{1 j}^{\prime} e^{-\lambda \tau_{1 j}^{\prime}}\right)^{-1} \\
= & \frac{P_{1}+i Q_{1}}{M_{1}+i N_{1}},
\end{aligned}
$$

where

$$
\begin{aligned}
P_{1}= & -A \cos \omega_{*} \tau_{2}-A \omega_{*} \tau_{2} \sin \omega_{*} \tau_{2}+B \tau_{2} \cos \omega_{*} \tau_{2} \\
& -C \cos \omega_{*} \tau_{1 j}^{\prime}+C \omega_{*} \tau_{1 j}^{\prime} \sin \omega_{*} \tau_{1 j}^{\prime} \\
& -E\left(\tau_{1 j}^{\prime}+\tau_{2}\right) \cos \omega_{*}\left(\tau_{1 j}^{\prime}+\tau_{2}\right), \\
Q_{1}= & 2 \omega_{*}+A \sin \omega_{*} \tau_{2}-A \omega_{*} \tau_{2} \cos \omega_{*} \tau_{2}-B \tau_{2} \sin \omega_{*} \tau_{2} \\
& +C \sin \omega_{*} \tau_{1 j}^{\prime}+C \omega_{*} \tau_{1 j}^{\prime} \cos \omega_{*} \tau_{1 j}^{\prime} \\
& +E\left(\tau_{1 j}^{\prime}+\tau_{2}\right) \sin \omega_{*}\left(\tau_{1 j}^{\prime}+\tau_{2}\right), \\
M_{1}= & E \omega_{*} \sin \omega_{*}\left(\tau_{1 j}^{\prime}+\tau_{2}\right)+C \omega_{*}^{2} \cos \omega_{*} \tau_{1 j}^{\prime}, \\
N_{1}= & E \omega_{*} \cos \omega_{*}\left(\tau_{1 j}^{\prime}+\tau_{2}\right)-C \omega_{*}^{2} \sin \omega_{*} \tau_{1 j}^{\prime} .
\end{aligned}
$$

Then

$$
\left(M_{1}^{2}+N_{1}^{2}\right)\left[\operatorname{Re}\left(\frac{d \lambda}{d \tau_{1}}\right)\right]_{\tau_{1}=\tau_{1 j}^{\prime}}^{-1}=M_{1} P_{1}+N_{1} Q_{1},
$$

noting that

$$
\left.\operatorname{Sign}\left[\operatorname{Re}\left(\frac{d \lambda}{d \tau_{1}}\right)\right]\right|_{\tau_{1}=\tau_{1_{0}}^{\prime}}=\left.\operatorname{Sign}\left[\operatorname{Re}\left(\frac{d \lambda}{d \tau_{1}}\right)^{-1}\right]\right|_{\tau_{1}=\tau_{1_{0}}^{\prime}}
$$

To obtain the transversal condition, we also need the condition as follows:

$$
M_{1} P_{1}+N_{1} Q_{1} \neq 0
$$

Case 5. $\tau_{1}$ is fixed in the interval $\left(0, \tau_{1_{0}}\right)$ and $\tau_{2}>0$.

Theorem 5. Assume that $\left(\mathrm{H}_{2}\right)$ holds; let $B+E>0$ and $\tau_{1} \in\left(0, \tau_{1_{0}}\right)$; then the equilibrium $E_{*}\left(x_{*}, y_{*}\right)$ is asymptotically stable for $\tau_{2} \in\left(0, \tau_{2_{0}}^{\prime}\right)$, and system (3) undergoes the Hopf bifurcation at $E_{*}\left(x_{*}, y_{*}\right)$ when $\tau_{2}=\tau_{2_{0}}^{\prime}$, where

$$
\begin{aligned}
\tau_{2_{0}}^{\prime}=\frac{1}{\omega_{0}} \arccos [ & \left(-C \omega_{0} \cos \omega_{0} \tau_{1}\left(A \omega_{0}+E \sin \omega_{0} \tau_{1}\right)\right. \\
& -\left(B-E \cos \omega_{0} \tau_{1}\right) \\
& \left.\times\left(\omega_{0}^{2}+C \omega_{0} \sin \omega_{0} \tau_{1}\right)\right) \\
& \times\left(\left(A \omega_{0}+E \sin \omega_{0} \tau_{1}\right)^{2}\right. \\
& \left.\left.\times\left(B-E \cos \omega_{0} \tau_{1}\right)^{2}\right)^{-1}\right],
\end{aligned}
$$

$$
M_{2} P_{2}+N_{2} Q_{2} \neq 0
$$

and $i \omega_{0}\left(\omega_{0}>0\right)$ is root of the corresponding characteristic equation; moreover

$$
\begin{aligned}
P_{2}= & -A \cos \omega_{0} \tau_{2}+A \omega_{0} \tau_{2} \sin \omega_{0} \tau_{2}+B \tau_{2} \cos \omega_{0} \tau_{2} \\
& -C \cos \omega_{0} \tau_{1}+C \omega_{0} \tau_{1} \sin \omega_{0} \tau_{1} \\
& -E\left(\tau_{1}+\tau_{2}\right) \cos \omega_{0}\left(\tau_{1}+\tau_{2}\right), \\
Q_{2}= & 2 \omega_{0}+A \sin \omega_{0} \tau_{2}+A \omega_{0} \tau_{2} \cos \omega_{0} \tau_{2}-B \tau_{2} \sin \omega_{0} \tau_{2} \\
& +C \sin \omega_{0} \tau_{1}+C \omega_{0} \tau_{1} \cos \omega_{0} \tau_{1} \\
& +E\left(\tau_{1}+\tau_{2}\right) \sin \omega_{0}\left(\tau_{1}+\tau_{2}\right), \\
M_{2}= & E \omega_{0} \sin \omega_{0}\left(\tau_{1}+\tau_{2}\right)+A \omega_{0}^{2} \cos \omega_{0} \tau_{1}-B \omega_{0} \sin \omega_{0} \tau_{2}, \\
N_{2}= & E \omega_{0} \cos \omega_{0}\left(\tau_{1}+\tau_{2}\right)-A \omega_{0}^{2} \sin \omega_{*} \tau_{1}-B \omega_{0} \cos \omega_{0} \tau_{2} .
\end{aligned}
$$

Proof. The proof is similar to that in Case 4.

\section{Direction and Stability of the Hopf Bifurcation}

In this section, we show that the system undergoes the Hopf bifurcation for different combinations of $\tau_{1}$ and $\tau_{2}$ satisfying sufficient conditions as described. Using the method based on the normal form theory and center manifold theory introduced by Hassard et al. in [13], we study the direction of bifurcations and the stability of bifurcating periodic solutions. Throughout this section, it is considered that the system undergoes the Hopf bifurcation at $\tau_{2}=\tau_{2_{0}}^{\prime}, \tau_{1} \in\left(0, \tau_{1_{0}}\right)$ at $E_{*}$. Let $\tau_{2}=\tau_{2_{0}}^{\prime}+\mu, \mu \in R$, so that the Hopf bifurcation occurs at $\mu=0$. Without loss of generality, it is assumed that $\tau_{1}^{*}<\tau_{2_{0}}^{\prime}$ where $\tau_{1}^{*} \in\left(0, \tau_{1_{0}}\right)$. Now we rescale the time by 
$t=t \tau_{2}, X(t)=x-x_{*}, Y(t)=y-y_{*} ;$ then system (3) can be written as

$$
\begin{aligned}
\dot{U}(t)=\left(\tau_{2_{0}}^{\prime}+\mu\right)( & B_{1} U(t)+B_{2} U\left(t-\frac{\tau_{1}^{*}}{\tau_{2}}\right) \\
& \left.+B_{3} U(t-1)+f(x, y)\right),
\end{aligned}
$$

where

$$
\begin{gathered}
U(t)=(X(t), Y(t))^{T}, \\
B_{1}=\left(\begin{array}{cc}
0 & \alpha_{1} \\
0 & 0
\end{array}\right), \quad B_{2}=\left(\begin{array}{cc}
\alpha_{2} & 0 \\
0 & 0
\end{array}\right), \\
B_{3}=\left(\begin{array}{cc}
0 & 0 \\
\alpha_{5} & \alpha_{6}
\end{array}\right), \quad f=\left(f_{1}, f_{2}\right)^{T} .
\end{gathered}
$$

For convenience, $X(t), Y(t)$ are still as $x(t), y(t)$, respectively; the nonlinear terms $f_{1}$ and $f_{2}$ are

$$
\begin{aligned}
& f_{1}=\alpha_{3} x(t) x\left(t-\frac{\tau_{1}^{*}}{\tau_{2}}\right)+\alpha_{4} x(t) y(t), \\
& f_{2}=\sum_{i+j+k \geq 2} \frac{1}{i ! j ! k !} f_{i j k} x^{i}(t-1) y^{j}(t-1) y^{k}(t) .
\end{aligned}
$$

Define a family of operators as

$$
\begin{aligned}
L_{\mu} \varphi & =\left(\tau_{2_{0}}^{\prime}+\mu\right)\left(B_{1} \varphi(0)+B_{2} \varphi\left(-\frac{\tau_{1}^{*}}{\tau_{2}}\right)+B_{3} \varphi(-1)\right), \\
\varphi & =\left(\varphi_{1}, \varphi_{2}\right)^{T} \in C\left([-1,0], R^{2}\right) .
\end{aligned}
$$

By the Riesz representation theorem, there exists a matrix whose components are bounded variation functions $\eta(\theta, \mu)$ : $[-1,0] \rightarrow R^{2}$ such that

$$
L_{\mu} \varphi=\int_{-1}^{0} d \eta(\theta, \mu) \varphi(\theta),
$$

where we choose

$$
\eta(\theta, \mu)= \begin{cases}\left(\tau_{2_{0}}^{\prime}+\mu\right)\left(B_{1}+B_{2}+B_{3}\right), & \theta=0, \\ \left(\tau_{2_{0}}^{\prime}+\mu\right)\left(B_{2}+B_{3}\right), & \theta \in\left[-\frac{\tau_{1}^{*}}{\tau_{2}}, 0\right), \\ \left(\tau_{2_{0}}^{\prime}+\mu\right) B_{3}, & \theta \in\left(-1,-\frac{\tau_{1}^{*}}{\tau_{2}}\right), \\ 0, & \theta=-1 .\end{cases}
$$

For $\varphi=\left(\varphi_{1}, \phi_{2}\right)^{T} \in C\left([-1,0], R^{2}\right)$, define

$$
\begin{aligned}
& A(\mu) \varphi= \begin{cases}\frac{d \varphi(\theta)}{d \theta}, & \theta \in[-1,0), \\
\int_{-1}^{0} d \eta(s, \mu) \varphi(s), & \theta=0,\end{cases} \\
& R(\mu) \varphi= \begin{cases}0, & \theta \in[-1,0), \\
h(\mu, \varphi), & \theta=0,\end{cases}
\end{aligned}
$$

where

$$
\begin{aligned}
& h(\mu, \varphi)=\left(\tau_{2_{0}}^{\prime}+\mu\right)\left(\begin{array}{l}
h_{1} \\
h_{2}
\end{array}\right), \\
& \varphi=\left(\varphi_{1}, \varphi_{2}\right)^{T} \in C\left([-1,0], R^{2}\right), \\
& h_{1}=\alpha_{3} \varphi_{1}(0) \varphi_{1}\left(-\frac{\tau_{1}^{*}}{\tau_{2}}\right)+\alpha_{4} \varphi_{1}(0) \varphi_{2}(0), \\
& h_{2}=\sum_{i+j+k \geq 2} \frac{1}{i ! j ! k !} f_{i j k} \varphi_{1}^{i}(-1) \varphi_{2}^{j}(-1) \varphi_{2}^{k}(0) .
\end{aligned}
$$

Hence, (3) can be rewritten as

$$
\dot{U}_{t}=A(\mu) U_{t}+R(\mu) U_{t}
$$

where $U=(X(t), Y(t))^{T}$ and $U_{t}(\theta)=U(t+\theta), \theta \in[-1,0]$. For $\psi \in C\left([0,1],\left(R^{2}\right)^{*}\right)$, define $A(0)=A$ and the adjoint operator $A^{*}$ of $A$ as

$$
A^{*} \psi(s)= \begin{cases}-\frac{d \psi(s)}{d s}, & s \in(0,1], \\ \int_{-1}^{0} d \eta^{T}(t, 0) \psi(-t), & s=0,\end{cases}
$$

where $\eta^{T}$ is the transpose of the matrix $\eta$.

For $\varphi \in C\left([-1,0], R^{2}\right)$ and $\psi \in C\left([0,1],\left(R^{2}\right)^{*}\right)$, in order to normalize the eigenvectors of operator $A$ and adjoint operator $A^{*}$, we define a bilinear inner product

$$
\begin{aligned}
\langle\psi(s), \varphi(\theta)\rangle= & \bar{\psi}(0) \varphi(0) \\
& -\int_{-1}^{0} \int_{\xi=0}^{\theta} \bar{\psi}(\xi-\theta) d \eta(\theta) \varphi(\xi) d \xi,
\end{aligned}
$$

where $\eta(\theta)=\eta(\theta, 0)$.

Since $\pm i \omega_{0} \tau_{20}^{\prime}$ are eigenvalues of $A$, they will also be the eigenvalues of $A^{*}$. The eigenvectors of $A$ and $A^{*}$ are calculated corresponding to the eigenvalues $+i \omega_{0} \tau_{2_{0}}^{\prime}$ and $-i \omega_{0} \tau_{2_{0}}^{\prime}$.

Lemma 6. $q(\theta)=(1, \rho)^{T} e^{i \omega_{0} \tau_{2_{0}}^{\prime} \theta}$ is the eigenvector of $A$ corresponding to $+i \omega_{0} \tau_{2_{0}}^{\prime} ; q^{*}(s)=(1 / D)(1, \sigma)^{T} e^{i \omega_{0} \tau_{2_{0}}^{\prime} s}$ is the eigenvector of $A^{*}$ corresponding to $-i \omega_{0} \tau_{2_{0}}^{\prime}$ and

$$
\left\langle q^{*}(s), q(\theta)\right\rangle=1, \quad\left\langle q^{*}(s), \bar{q}(\theta)\right\rangle=0,
$$

where

$$
\begin{gathered}
\rho=\frac{i \omega_{0}-\alpha_{2} e^{-i \omega_{0}\left(\tau_{1}^{*} / \tau_{2_{0}}^{\prime}\right)}}{\alpha_{1}}, \quad \sigma=\frac{-i \omega_{0}+\alpha_{2} e^{-i \omega_{0} \tau_{2_{0}}^{\prime}}}{\alpha_{5} e^{i \omega_{0} \tau_{2_{0}}^{\prime}}}, \\
\bar{D} \\
=1+\rho \bar{\sigma}+\tau_{2_{0}}^{\prime}\left[\left(\alpha_{5} \bar{\sigma}+\alpha_{6} \rho \bar{\sigma}\right) e^{-i \omega_{0} \tau_{2_{0}}^{\prime}}+\frac{\tau_{1}^{*}}{\tau_{2_{0}}^{\prime}} \alpha_{2} e^{-i \omega_{0}\left(\tau_{1}^{*} / \tau_{2_{0}}^{\prime}\right)}\right] .
\end{gathered}
$$$$
\bar{D}
$$ 
Following the algorithms explained in Hassard et al. [13], we can obtain the properties of the Hopf bifurcation:

$$
\begin{aligned}
& g_{20}=\frac{2 \tau_{2_{0}}^{\prime}}{\bar{D}}\left[\alpha_{3} e^{-i \omega_{0}\left(\tau_{1}^{*} / \tau_{20}^{\prime}\right)}+\alpha_{4} \rho\right. \\
& +\bar{\sigma}\left(\frac{1}{2} f_{200} e^{-2 i \omega_{0} \tau_{20}^{\prime}}+f_{110} \rho e^{-2 i \omega_{0} \tau_{20}^{\prime}}\right. \\
& \left.\left.+f_{101} \rho e^{-2 i \omega_{0} \tau_{20}^{\prime}}+f_{011} \rho^{2} e^{-i \omega_{0} \tau_{20}^{\prime}}\right)\right], \\
& g_{11}=\frac{\tau_{2_{0}}^{\prime}}{\bar{D}}\left[2 \alpha_{3} e^{-i \omega_{0}\left(\tau_{1}^{*} / \tau_{20}^{\prime}\right)}+\alpha_{4}(\bar{\rho}+\rho)\right. \\
& +\bar{\sigma}\left(f_{200}+f_{110}(\bar{\rho}+\rho)+f_{101} \rho e^{i \omega_{0} \tau_{20}^{\prime}}\right. \\
& \left.\left.+f_{101} \bar{\rho} e^{-i \omega_{0} \tau_{20}^{\prime}}+2 f_{011} \bar{\rho} \rho e^{-i \omega_{0} \tau_{20}^{\prime}}\right)\right], \\
& g_{02}=\frac{2 \tau_{2_{0}}^{\prime}}{\bar{D}}\left[\alpha_{3} e^{-i \omega_{0}\left(\tau_{1}^{*} / \tau_{20}^{\prime}\right)}+\alpha_{4}(\bar{\rho}+\rho)\right. \\
& +\bar{\sigma}\left(\frac{1}{2} f_{200} e^{2 i \omega_{0} \tau_{20}^{\prime}}+f_{110} \bar{\rho} e^{2 i \omega_{0} \tau_{20}^{\prime}}\right. \\
& \left.\left.+f_{101} \bar{\rho} e^{i \omega_{0} \tau_{20}^{\prime}}+f_{011} \bar{\rho}^{2} \rho e^{i \omega_{0} \tau_{20}^{\prime}}\right)\right], \\
& g_{21}=\frac{2 \tau_{2_{0}}^{\prime}}{\bar{D}}\left\{\alpha _ { 3 } \left(W_{11}^{(1)}\left(\frac{\tau_{1}^{*}}{\tau_{20}^{\prime}}\right)+\frac{1}{2} W_{20}^{(1)}\left(\frac{\tau_{1}^{*}}{\tau_{20}^{\prime}}\right)\right.\right. \\
& +\frac{1}{2} W_{20}^{(1)}(0) e^{-i \omega_{0}\left(\tau_{1}^{*} / \tau_{20}^{\prime}\right)} \\
& \left.+W_{11}^{(1)}(0) e^{-i \omega_{0}\left(\tau_{1}^{*} / \tau_{20}^{\prime}\right)}\right) \\
& +\alpha_{4}\left(W_{11}^{(2)}(0)+\frac{1}{2} W_{20}^{(2)}(0)\right. \\
& \left.+\frac{1}{2} W_{20}^{(1)}(0) \bar{\rho}+\rho W_{11}^{(1)}(0)\right) \\
& +\bar{\sigma}\left[\frac { 1 } { 2 } f _ { 2 0 0 } \left(2 W_{11}^{(1)}(-1) e^{-i \omega_{0} \tau_{2_{0}}^{\prime}}\right.\right. \\
& \left.+W_{20}^{(1)}(-1) e^{i \omega_{0} \tau_{2_{0}}^{\prime}}\right) \\
& +f_{110}\left(W_{11}^{(1)}(-1) e^{-i \omega_{0} \tau_{20}^{\prime}}\right. \\
& +\frac{1}{2} W_{20}^{(2)}(-1) e^{i \omega_{0} \tau_{2_{0}}^{\prime}} \\
& +\frac{1}{2} W_{20}^{(1)}(-1) \bar{\rho} e^{i \omega_{0} \tau_{2_{0}}^{\prime}} \\
& \left.+W_{11}^{(1)}(-1) \rho e^{-i \omega_{0} \tau_{2_{0}}^{\prime}}\right)
\end{aligned}
$$

$$
\begin{aligned}
&+f_{101}( W_{11}^{(1)}(-1) \rho+\frac{1}{2} W_{20}^{(1)}(-1) \bar{\rho} \\
&+\frac{1}{2} W_{20}^{(2)}(0) e^{i \omega_{0} \tau_{2_{0}}^{\prime}} \\
&\left.+W_{11}^{(2)}(0) e^{-i \omega_{0} \tau_{2_{0}}^{\prime}}\right) \\
&+f_{011}\left(W_{11}^{(2)}(-1) \rho+\frac{1}{2} W_{20}^{(2)}(-1) \bar{\rho}\right. \\
&+\frac{1}{2} W_{20}^{(2)}(0) \bar{\rho} e^{i \omega_{0} \tau_{2_{0}}^{\prime}} \\
&+W_{11}^{(2)}(0) \rho e^{-i \omega_{0} \tau_{2_{0}}^{\prime}} \\
&\left.\left.\left.+\frac{1}{2} f_{300} e^{i \omega_{0} \tau_{2_{0}}^{\prime}}\right)\right]\right\},
\end{aligned}
$$

where

$$
\begin{aligned}
W_{20}(\theta)= & \frac{i g_{20}}{\omega_{0} \tau_{2_{0}}^{\prime}} q(0) e^{i \theta \omega_{0} \tau_{2_{0}}^{\prime}} \\
& +\frac{i \bar{g}_{02}}{3 \tau_{2_{0}}^{\prime} \omega_{0}} \bar{q}(0) e^{-i \theta \omega_{0} \tau_{2_{0}}^{\prime}}+\operatorname{Re}^{2 i \theta \omega_{0} \tau_{2_{0}}^{\prime}} \\
W_{11}(\theta)=- & \frac{i g_{11}}{\tau_{2_{0}}^{\prime} \omega_{0}} q(0) e^{i \theta \omega_{0} \tau_{2_{0}}^{\prime}} \\
& +\frac{i \bar{g}_{11}}{\tau_{2_{0}}^{\prime} \omega_{0}} \bar{q}(0) e^{-i \theta \omega_{0} \tau_{2_{0}}^{\prime}}+S .
\end{aligned}
$$

We know that $R=\left(R^{(1)}, R^{(2)}\right) \in R^{2}$ and $S=\left(S^{(1)}, S^{(2)}\right) \in R^{2}$ are constant vectors, computed as

$$
\begin{aligned}
& R \\
= & 2\left(\begin{array}{cc}
2 i \omega_{0}-\alpha_{2} e^{-2 i \omega_{0}\left(\tau_{1}^{*} / \tau_{20}^{\prime}\right)} & -\alpha_{1} \\
-\alpha_{5} e^{-2 i \omega_{0} \tau_{20}^{\prime}} & 2 i \omega_{0}-\alpha_{6} e^{-2 i \omega_{0} \tau_{20}^{\prime}}
\end{array}\right)^{-1} \\
& \times\left(\begin{array}{c}
\left(\begin{array}{c}
1 \\
\frac{1}{2} f_{200}+f_{110} \rho+f_{101} \rho
\end{array}\right) e^{-2 i \omega_{0} \tau_{20}^{\prime}}+f_{011} \rho^{2} e^{-i \omega_{0} \tau_{20}^{\prime}}
\end{array}\right), \\
S & \left(\begin{array}{cc}
-\alpha_{2}\left(\tau_{1}^{*} / \tau_{20}^{\prime}\right) & -\alpha_{1} \\
-\alpha_{5} & -\alpha_{6}
\end{array}\right) \\
& \times\left(\begin{array}{c}
-1 \\
2 \alpha_{3} e^{-i \omega_{0}\left(\tau_{1}^{*} / \tau_{20}^{\prime}\right)}+\alpha_{4}(\bar{\rho}+\rho) \\
f_{200}+f_{110}(\bar{\rho}+\rho)+f_{101} \rho e^{i \omega_{0} \tau_{20}^{\prime}}+\left(f_{101} \bar{\rho}+2 f_{011} \bar{\rho} \rho\right) e^{-i \omega_{0} \tau_{20}^{\prime}}
\end{array}\right) .
\end{aligned}
$$

As a result, we know $W_{20}(\theta)$ and $W_{11}(\theta)$; then $g_{i j}$ is determined by the parameters and delays $\tau_{2_{0}}^{\prime}$ and $\tau_{1}^{*}$. Thus, we can compute the following quantities:

$$
\begin{gathered}
c_{1}(0)=\frac{i}{2 \omega_{0} \tau_{2_{0}}^{\prime}}\left(g_{20} g_{11}-2\left|g_{11}\right|^{2}-\frac{1}{3}\left|g_{02}\right|^{2}\right)+\frac{g_{21}}{2}, \\
\mu_{2}=-\frac{\operatorname{Re}\left\{c_{1}(0)\right\}}{\operatorname{Re}\left\{\lambda^{\prime}\left(\tau_{2_{0}}^{\prime}\right)\right\}},
\end{gathered}
$$




$$
\begin{gathered}
\beta_{2}=2 \operatorname{Re}\left\{c_{1}(0)\right\}, \\
T_{2}=-\frac{\operatorname{Im}\left\{c_{1}(0)\right\}+\mu_{2} \operatorname{Im}\left\{\lambda^{\prime}\left(\tau_{2_{0}}^{\prime}\right)\right\}}{\omega_{0} \tau_{2_{0}}^{\prime}} .
\end{gathered}
$$

These expressions give a description of the bifurcating periodic solutions in the center manifold of system (3) at critical values $\tau_{2}=\tau_{2_{0}}^{\prime}$ and when $\operatorname{Re}\left\{\lambda^{\prime}\left(\tau_{2_{0}}^{\prime}\right)\right\}>0$ which can be stated as follows:

(i) $\mu_{2}$ gives the direction of the Hopf bifurcation: if $\mu_{2}>$ $0\left(\mu_{2}<0\right)$, the Hopf bifurcation is supercritical (subcritical);

(ii) $\beta_{2}$ determines the stability of bifurcating periodic solution: the periodic solutions are stable (unstable) if $\beta_{2}<0\left(\beta_{2}>0\right)$;

(iii) $T_{2}$ denotes the period of bifurcating period solutions: if $T_{2}>0\left(T_{2}<0\right)$, periodic solutions increase (decrease).

\section{Numerical Simulations}

To demonstrate the algorithm for determining the existence of the Hopf bifurcation in Section 2 and the direction and stability of the Hopf bifurcation in Section 3, we carry out numerical simulations on a particular case of (3) in the following form:

$$
\begin{aligned}
& \dot{x}(t)=0.8 x(t)\left(1-\frac{x\left(t-\tau_{1}\right)}{0.7}\right)-1.3(1-0.5) x(t) y(t), \\
& \dot{y}(t)=y(t)\left(1-\frac{y\left(t-\tau_{2}\right)}{(1-0.5) x\left(t-\tau_{2}\right)}\right),
\end{aligned}
$$

where $r_{1}=0.8, r_{2}=1, a=1.3, K=0.7, \gamma=1$, and $m=$ 0.5 . It is easy to show that system (55) has unique coexistence equilibrium $E_{*}(0.545,0.2725)$. By calculation, when $\tau_{1}=0$, the critical delay for $\tau_{2}$ is obtained as $\tau_{2_{0}}=1.3507$ and $\tau_{1_{0}}=$ 5.8228 when $\tau_{2}=0$.

We can see from Figure 1(a) that $E_{*}$ is asymptotically stable at $\tau_{1}=0, \tau_{2}=1.1<\tau_{2_{0}}=1.3507$, while from Figure 1(b) $E_{*}$ loses stability and the Hopf bifurcation occurs at $\tau_{1}=0, \tau_{2}=1.5>\tau_{2_{0}}=1.3507$. From Figure 2(a), $E_{*}$ is asymptotically stable when $\tau_{1}=2.8<\tau_{1_{0}}=5.8228, \tau_{2}=$ 0 , while from Figure 2(b) $E_{*}$ loses stability and the Hopf bifurcation occurs when $\tau_{1}=6.5>\tau_{1_{0}}=5.8228, \tau_{2}=0$.

Further, under the condition of $\tau_{1}=1.28$, when $\tau_{2}=$ $1.32<\tau_{2_{0}}^{\prime}=1.9507, E_{*}$ is also stable (see Figure 3(a)), while, at $\tau_{2}=5.83, E_{*}$ loses stability and the Hopf bifurcation occurs from Figure 3(b); then using the algorithm derived in Section 3, we obtain that $\mu_{2}=312.8, \beta_{2}=-287.5$, $T_{2}=106.56$; we know the Hopf bifurcation is supercritical and bifurcating periodic solutions are stable and increase. When $\tau_{2}=7.9$, system (55) becomes a chaotic solution in Figure 3(c). In Figure 3(d), the largest Lyapunov exponent diagram is plotted for variable $\tau_{2}$; it is easy to know that when $\tau_{2}>7.55$, the Lyapunov exponent is almost positive; then the chaos occurs.

Whereas, when $\tau_{1}=10.15>\tau_{1_{0}}=5.8228$ and $\tau_{2}=1.2$, system (55) becomes chaotic in Figure 4(a), in Figure 4(b), the largest Lyapunov exponent diagram is plotted for variable $\tau_{1}$; it is easy to know that when $\tau_{1}>9.85$, the Lyapunov exponent is almost positive; then the chaotic solutions occur.

However, $E_{*}$ loses stability and the Hopf bifurcation occurs at $\tau_{1}=6.9, \tau_{2}=2.1$ in Figure 5(a). When $\tau_{1}=$ 9.3, $\tau_{2}=2.7$, a chaotic solution occurs in Figure 5(b). To explore the possibility of occurrence of chaos, the largest Lyapunov exponent diagrams are plotted with respect to key parameters $\tau_{1}$ and $\tau_{2}$. In Figure 5(c), the largest Lyapunov exponent diagram is plotted for variable $\tau_{1}$ when $\tau_{2}=2.7$; it is easy to know that when $\tau_{1}>9.15$, the Lyapunov exponent is almost positive; then the chaotic solution occurs. Similarly, in Figure 5(d), the largest Lyapunov exponent diagram is plotted for variable $\tau_{2}$ when $\tau_{1}=9.3$; it is easy to know that when $\tau_{2}>2.65$, the Lyapunov exponent is almost positive; then the chaotic solution occurs.

\section{Global Continuation of the Local Hopf Bifurcation}

In this section, we will study the global continuation of periodic solutions bifurcating from the point $E_{*}$ for $\tau_{1}$ is fixed in the interval $\left(0, \tau_{1}\right)$. Further, the method we used here is based on the global Hopf bifurcating theorem for general functional differential equations introduced by $\mathrm{Wu}$ [14]. For convenience, we denote $\tau=\tau_{2}$ and write system (3) in the following form:

$$
\dot{z}(t)=F\left(z_{t}, \tau, p\right),
$$

where $z_{t}=\left(z_{1 t}(\theta), z_{2 t}(\theta)\right)^{T}=\left(z_{1}(t+\theta), z_{2}(t+\theta)\right)^{T} \epsilon$ $C\left([-\tau, 0], R^{2}\right)$. Following the work of $\mathrm{Wu}[14]$, we define

$$
\begin{aligned}
& X=C\left([-\tau, 0], R^{2}\right), \\
& \Sigma=C l\left\{(z(t), \tau, p) \in X \times R^{+} \times\right. \\
& \text {solution of }(56)\}, \\
& N=\{(\bar{z}, \tau, p), F(\bar{z}, \tau, p)=0\} .
\end{aligned}
$$$$
\Sigma=C l\left\{(z(t), \tau, p) \in X \times R^{+} \times R^{+}, z(t) \text { is a } p\right. \text {-periodic }
$$

Lemma 7. Assume that $(\bar{z}, \tau, p)$ is an isolated center satisfying $(A 1-A 4)$ in [14]. Denote by $l_{(\bar{z}, \tau, p)}$ the connected component of $(\bar{z}, \tau, p)$ in $\Sigma$. Then either

(i) $l_{(\bar{z}, \tau, p)}$ is unbounded or

(ii) $l_{(\bar{z}, \tau, p)}$ is bounded, $l_{(\bar{z}, \tau, p)} \cap N$ is finite, and $\sum_{(\bar{z}, \tau, p) \in l_{\bar{z}, \tau, p} \cap N} \gamma_{m}(\bar{z}, \tau, p)=0$ for all $m=1,2,3, \ldots$, where $\gamma_{m}(\bar{z}, \tau, p)$ is the mth crossing number of $(\bar{z}, \tau, p)$, if $m \in J(\bar{z}, \tau, p)$, or it is zero otherwise.

It is well known that if (ii) of the theorem is not true, then $l_{(\bar{z}, \tau, p)}$ is unbounded. However, when the projections of $l_{(\bar{z}, \tau, p)}$ onto $z$-space and onto $p$-space are bounded, then the projection of $l_{(\bar{z}, \tau, p)}$ onto $\tau$-space is unbounded. Further, we show that the projection of $l_{(\bar{z}, \tau, p)}$ onto $\tau$-space is away from zero; then the projection of $\tau$-space must include $[\tau, \infty)$. 


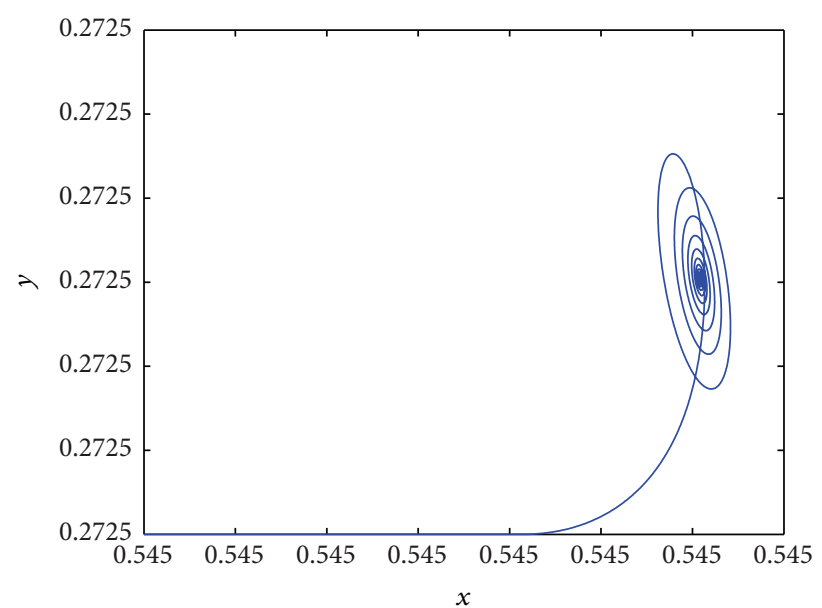

(a)

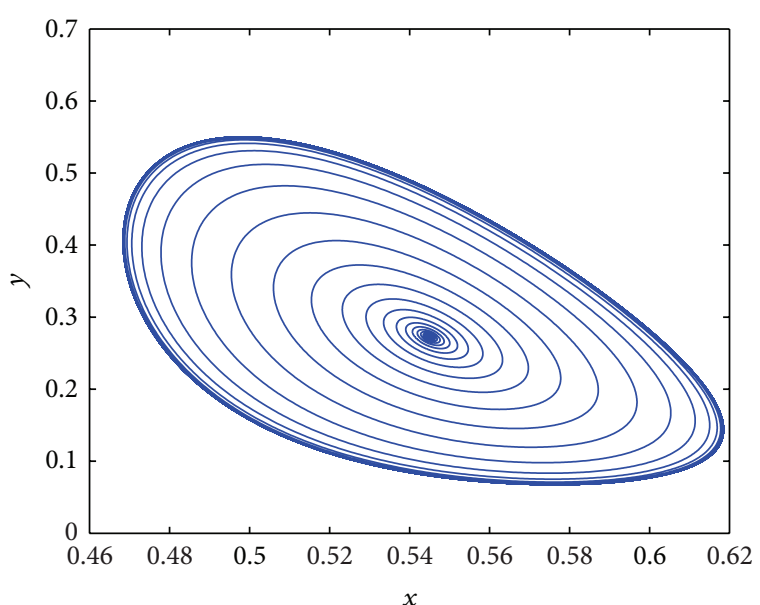

(b)

Figure 1: (a) $E_{*}$ is asymptotically stable equilibrium at $\tau_{1}=0$ and $\tau_{2}=1.1$; (b) $E_{*}$ loses stability and the Hopf bifurcation occurs at $\tau_{1}=$ $0, \tau_{2}=1.5$.

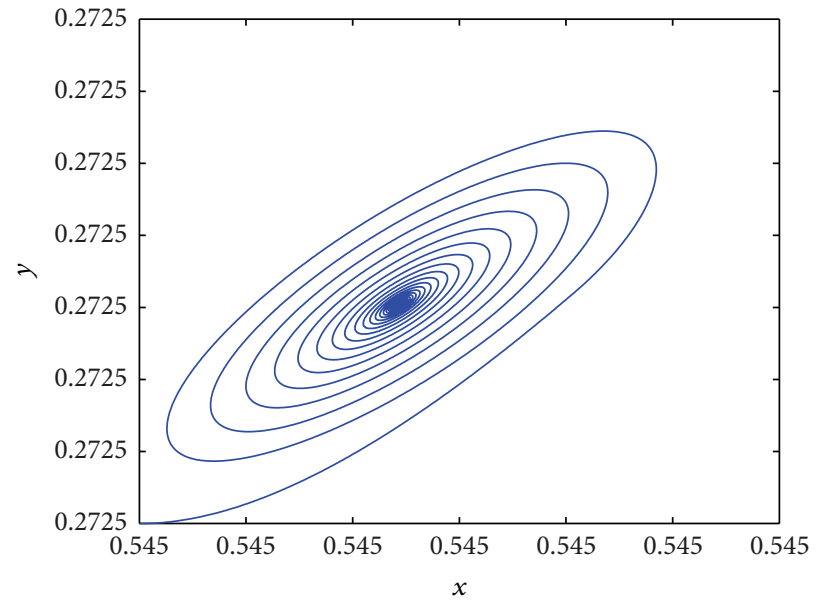

(a)

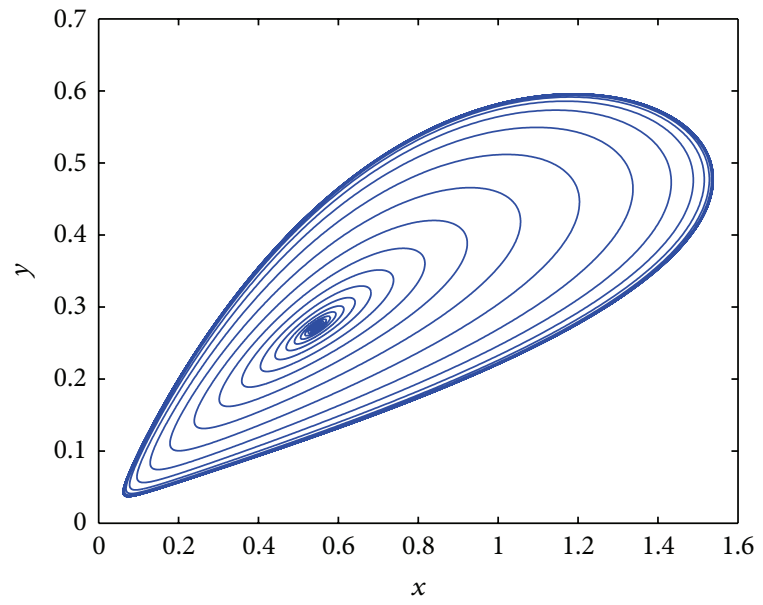

(b)

FIGURE 2: (a) $E_{*}$ is asymptotically stable equilibrium at $\tau_{1}=2.8$ and $\tau_{2}=0$; (b) $E_{*}$ loses stability and the Hopf bifurcation occurs at $\tau_{1}=$ $6.5, \tau_{2}=0$.

Following this idea, we can prove our results on the global continuation of the local Hopf bifurcation.

Lemma 8. If $\left(\mathrm{H}_{2}\right)$ and $B+E>0$ hold, nontrivial periodic solutions of (3) are uniformly bounded.

Proof. Let $x(t), y(t)$ be a nontrivial solution of system (3) through $(\varphi, \psi)$ at $t=0$ with $\varphi(0)>0, \psi(0)>0$. Then it follows from (3) that

$$
\begin{aligned}
x(t)=x(0) \exp \int_{0}^{t} & \left(r_{1}-\frac{r_{1} x\left(s-\tau_{1}\right)}{K}\right. \\
& -a(1-m) x(s) y(s)) d s,
\end{aligned}
$$

$$
y(t)=y(0) \exp \int_{0}^{t}\left(r_{2}-\frac{r_{2} y(s-\tau)}{\gamma(1-m) x(s-\tau)}\right) d s,
$$

which implies that solutions of system (3) cannot cross the $x$ axes and $y$-axes. Thus, the nontrivial periodic orbits must be located in the interior of the first quadrant.

Since $(x(t), y(t))$ is a nontrivial solution of (3) with $x(t)>$ $0, y(t)>0$, then we have

$$
\dot{x}(t)<r_{1} x(t)\left(1-\frac{x\left(t-\tau_{1}\right)}{K}\right) .
$$




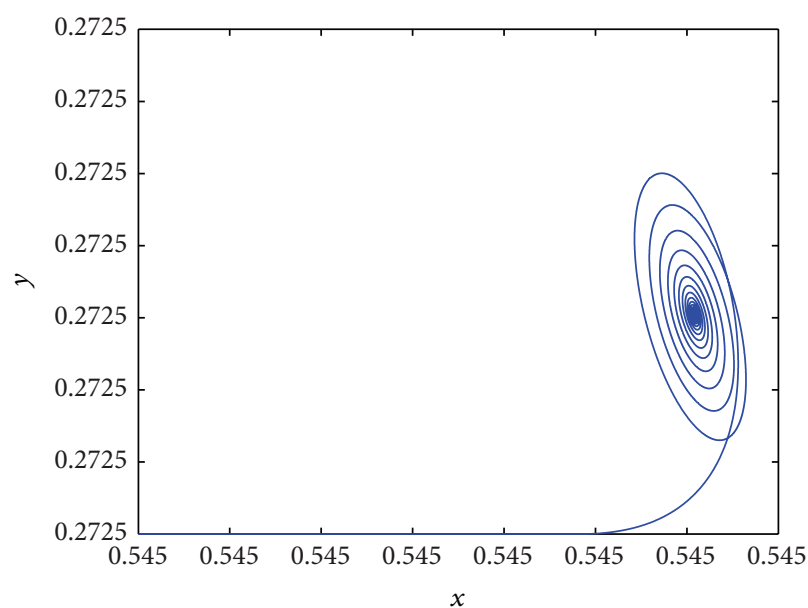

(a)

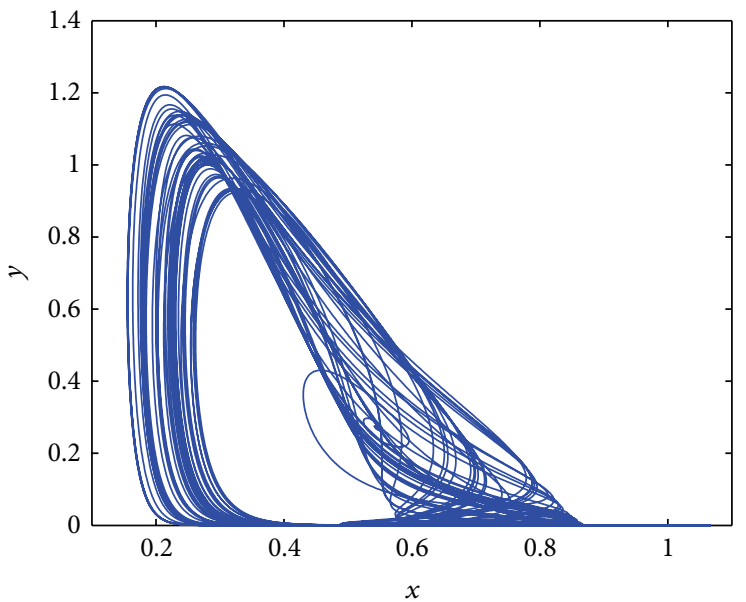

(c)

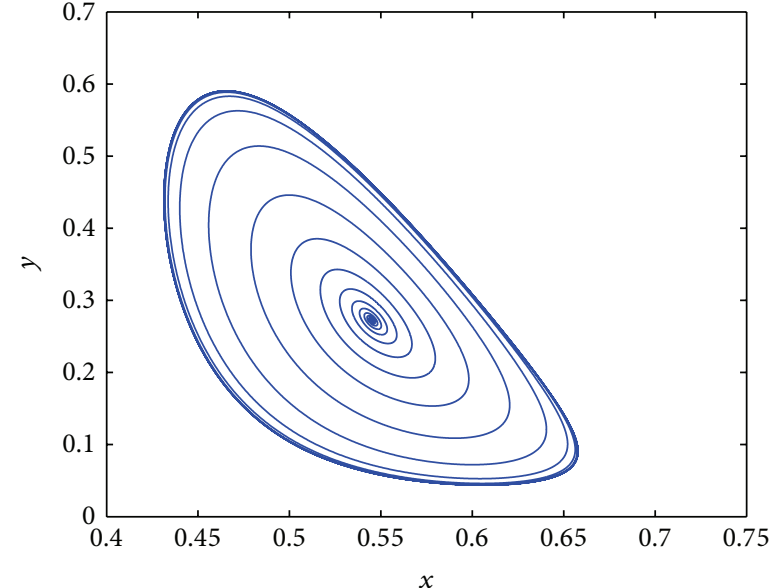

(b)

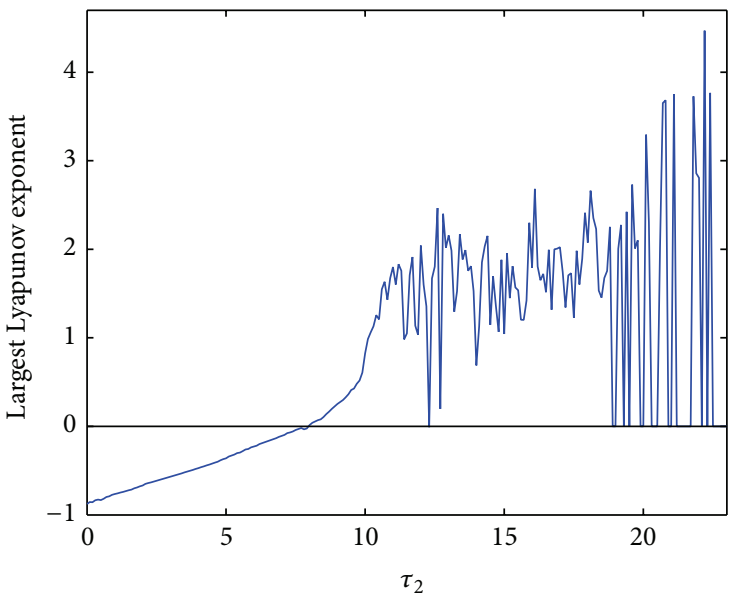

(d)

FIGURE 3: Under the condition of $\tau_{1}=1.28$, (a) $E_{*}$ is asymptotically stable equilibrium at $\tau_{2}=1.32$; (b) $E_{*}$ loses stability and the Hopf bifurcation occurs at $\tau_{2}=5.83$; (c) a chaotic solution occurs at $\tau_{2}=7.9$; (d) the largest Lyapunov exponent diagram of system (55) for variable $\tau_{2}$.

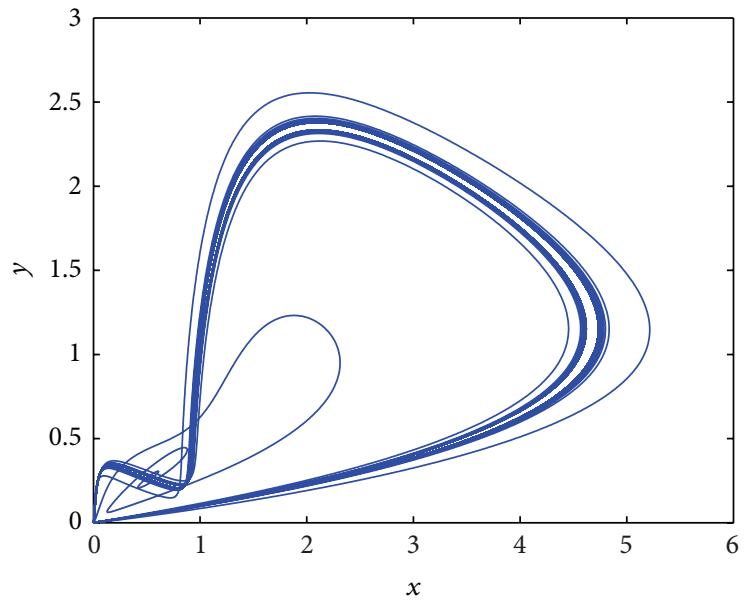

(a)

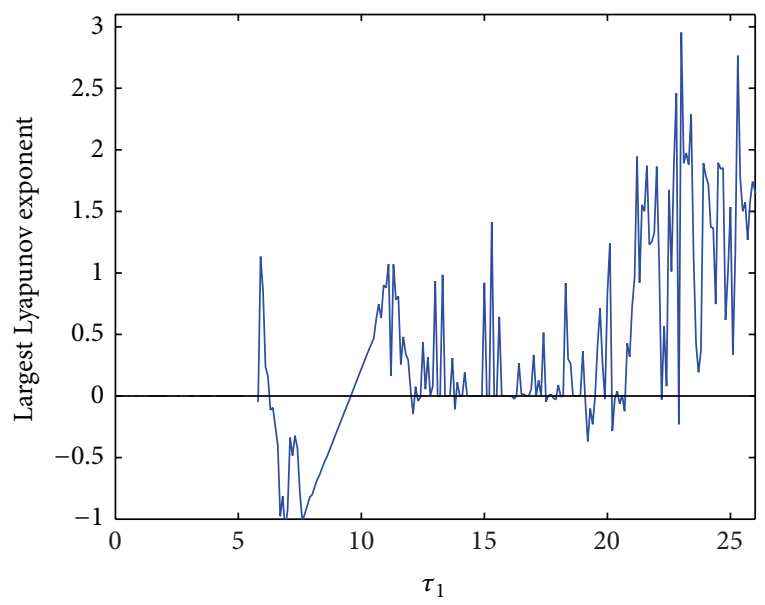

(b)

Figure 4: (a) A chaotic solution occurs at $\tau_{1}=10.15, \tau_{2}=1.2$; (b) the largest Lyapunov exponent diagram of system (55) for variable $\tau_{1}$. 


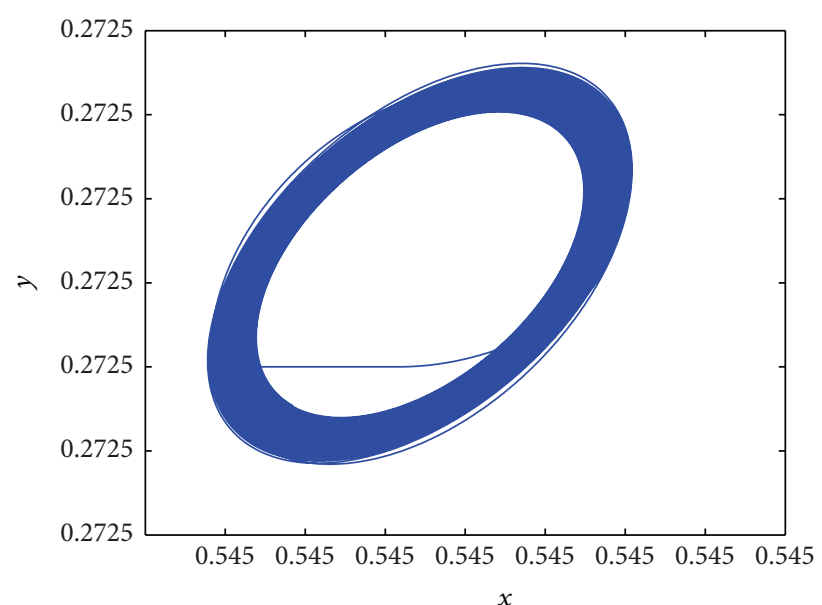

(a)

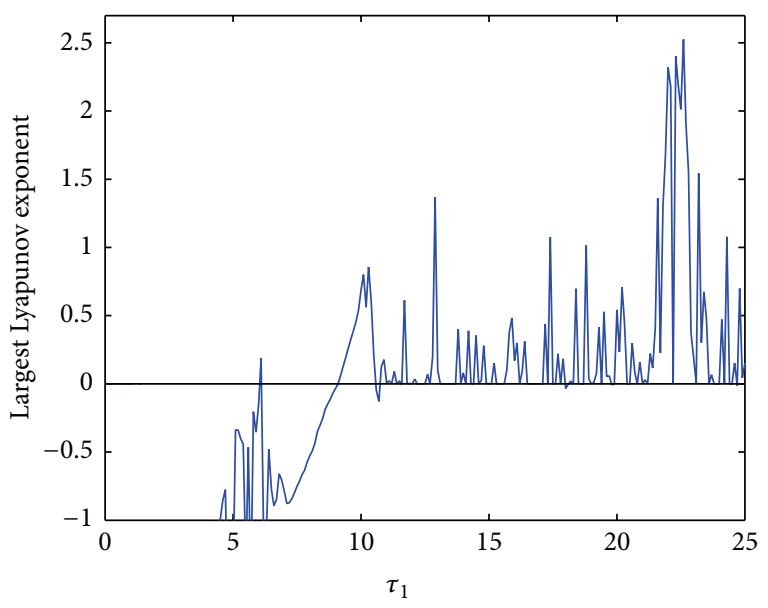

(c)

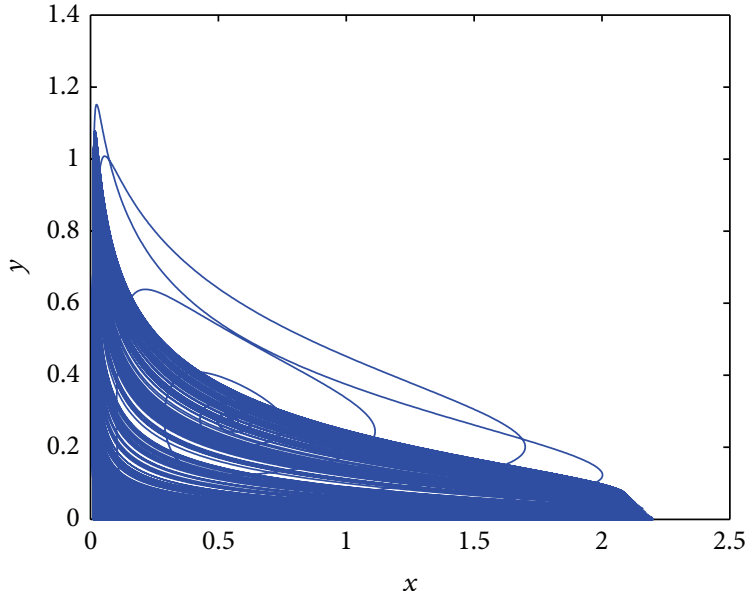

(b)

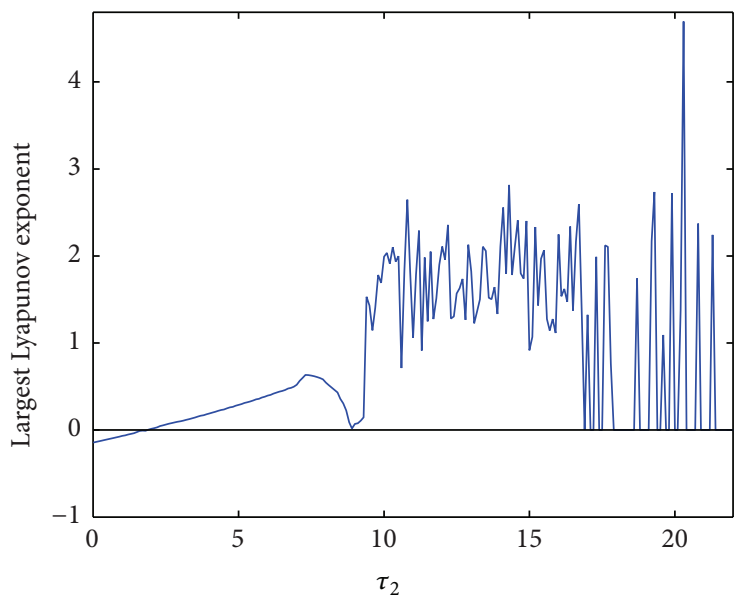

(d)

Figure 5: (a) The Hopf bifurcation occurs at $\tau_{1}=6.9, \tau_{2}=2.1$; (b) chaotic solution occurs at $\tau_{1}=9.3, \tau_{2}=2.7$; (c) the largest Lyapunov exponent diagram of system (55) for variable $\tau_{1}$ at $\tau_{2}=2.7$; (d) the largest Lyapunov exponent diagram of system (55) for variable $\tau_{2}$ at $\tau_{1}=9.3$.

It is easy to know $x(t)<x\left(t-\tau_{1}\right) e^{r_{1} \tau_{1}}$ for $t>\tau_{1}$; then if $t>\tau_{1}$, we obtain

$$
\dot{x}(t)<r_{1} x(t)\left(1-\frac{x(t) e^{-r_{1} \tau_{1}}}{K}\right),
$$

which implies that

$$
\lim \sup _{t \rightarrow \infty} x(t) \leq K e^{r_{1} \tau_{1}} .
$$

Thus, for any $\varepsilon>0$, there exists a $T>0$ such that when $t>T$, we have

$$
x(t) \leq K e^{r_{1} \tau_{1}}+\varepsilon:=\delta .
$$

It follows from the second equation of (3) that, for $t>T+\tau$,

$$
\dot{y}(t)<r_{2} y(t)\left[1-\frac{y(t-\tau)}{\gamma(1-m) \delta}\right] .
$$

Clearly, $y(t)<\gamma(1-m) \delta e^{r_{1} \tau}$ for sufficiently large $t$. Thus, the nontrivial periodic solutions lying in the first quadrant of system (3) must be uniformly bounded.
Lemma 9. If $\left(\mathrm{H}_{2}\right)$ and $B+E>0$ hold, system (3) has no nontrivial periodic solutions with period $\tau$.

Proof. Assume that system (3) has a nontrivial periodic solution of period $\tau$; then the differential system

$$
\begin{aligned}
& \dot{x}(t)=r_{1} x(t)\left(1-\frac{x\left(t-\tau_{1}\right)}{K}\right)-a(1-m) x(t) y(t), \\
& \dot{y}(t)=r_{2} y(t)\left(1-\frac{y(t)}{\gamma(1-m) x(t)}\right),
\end{aligned}
$$

has periodic solution with period $\tau$. Due to Lemma 7, we restrict our attention to $0<x(t)<K, 0<y(t)<\gamma K(1-$ $m)$, respectively. System (63) also has the equilibrium $E_{*}=$ $\left(x_{*}, y_{*}\right)$; we define

$$
V(x, y)=\ln \frac{x}{x_{*}}+\frac{x_{*}}{x}+\frac{a x_{*} \gamma(1-m)^{2}}{r_{2}}\left(\ln \frac{y}{y_{*}}+\frac{y_{*}}{y}\right) .
$$


Obviously, $V(x, y)$ is well defined and continuous for all $x(t)>0, y(t)>0$. The function $V(x, y)$ satisfies

$$
\frac{\partial V}{\partial x}=\frac{1}{x}\left(1-\frac{x_{*}}{x}\right), \quad \frac{\partial V}{\partial y}=\frac{a x_{*} \gamma(1-m)^{2}}{r_{2} y}\left(1-\frac{y_{*}}{y}\right) .
$$

Equation (65) shows that the positive equilibrium $\left(x_{*}, y_{*}\right)$ is the only extremum of the function $V(x, y)$ in the first quadrant. It is easy to see that the point $\left(x_{*}, y_{*}\right)$ is a minimum, since

$$
\begin{aligned}
\lim _{x \rightarrow 0} V(x, y) & =\lim _{y \rightarrow 0} V(x, y)=\lim _{x \rightarrow \infty} V(x, y) \\
& =\lim _{y \rightarrow \infty} V(x, y)=+\infty .
\end{aligned}
$$

Clearly, the positive equilibrium $\left(x_{*}, y_{*}\right)$ is the global minimum; that is,

$$
V(x, y)>V\left(x_{*}, y_{*}\right)=1+\frac{a x_{*} \gamma(1-m)^{2}}{r_{2}}>0
$$

holds for all $x(t)>0, y(t)>0$.

Calculate the derivative of $V$ along the solution of system (3). Use Razumikhin's theorem (see [15]); when $0<x\left(t-\tau_{1}\right)<$ $x(t)$, we have

$$
\frac{d V}{d t}<-\frac{r_{1}}{K x}\left(x\left(t-\tau_{1}\right)-x_{*}\right)^{2}-\frac{a(1-m)}{y}\left(y-y_{*}\right)^{2}<0 .
$$

Thus, $V(x, y)$ satisfies Lyapunov's asymptotic stability theorem; we conclude that

$$
\lim _{t \rightarrow \infty}(x(t), y(t))=\left(x_{*}, y_{*}\right)
$$

which contradicts the fact that system (63) has periodic solutions. This ends the proof.

Theorem 10. If $\left(\mathrm{H}_{2}\right)$ and $B+E>0$ hold, let $\omega_{0}$ and $\tau_{2_{j}}^{\prime}(j=$ $0,1,2, \ldots)$ be defined in Case 5 in Section 2. Then, for each $\tau>$ $\tau_{2_{j}}^{\prime}(j \geq 1)$, system (3) has at least $j+1$ periodic solutions.

Proof. It is easy to know that the characteristic matrix of system (3) at the positive equilibrium $z^{*}$ is of the form

$$
\Delta\left(z^{*}, \tau, p\right)(\lambda)=\left(\begin{array}{cc}
\lambda-\alpha_{2} e^{-\lambda \tau_{1}} & -\alpha_{1} \\
-\alpha_{5} e^{-\lambda \tau} & \lambda-\alpha_{6} e^{-\lambda \tau}
\end{array}\right)
$$

From the discussion of Section 2, it can be verified that $\left(z^{*}, \tau_{2}^{\prime}, 2 \pi / \omega_{0}\right), j=1,2, \ldots$, are isolated centers.

Let

$$
\Omega_{\epsilon, 2 \pi / \omega_{0}}=\left\{(\eta, p): 0<\eta<\epsilon,\left|p-\frac{2 \pi}{\omega_{0}}\right|<\epsilon\right\} .
$$

Clearly, if $\left|\tau-\tau_{2_{j}}^{\prime}\right| \leq \delta$ and $(\eta, p) \in \partial \Omega_{\epsilon}$, then the necessary and sufficient conditions for $\operatorname{det}\left(\Delta\left(z^{*}, \tau, p\right)(\eta+i(2 \pi / p))\right)=0$ are $\eta=0, \tau=\tau_{2_{j}}^{\prime}$, and $p=2 \pi / \omega_{0}$.

Defining

$$
\begin{aligned}
& H^{ \pm}\left(z^{*}, \tau_{2_{j}}^{\prime}, \frac{2 \pi}{\omega_{0}}\right)(\eta, p) \\
& \quad=\operatorname{det}\left(\Delta\left(z^{*}, \tau_{2_{j}}^{\prime} \pm \delta, p\right)\left(\eta+i \frac{2 \pi}{p}\right)\right),
\end{aligned}
$$

then we have the transversal number

$$
\begin{aligned}
\gamma\left(z^{*}, \tau_{2_{j}}^{\prime}, \frac{2 \pi}{\omega_{0}}\right)= & \operatorname{deg}_{B}\left(H^{-}\left(z^{*}, \tau_{2_{j}}^{\prime}, \frac{2 \pi}{\omega_{0}}\right), \Omega_{\epsilon, 2 \pi / \omega_{0}}\right) \\
& -\operatorname{deg}_{B}\left(H^{+}\left(z^{*}, \tau_{2_{j}}^{\prime}, \frac{2 \pi}{\omega_{0}}\right), \Omega_{\epsilon, 2 \pi / \omega_{0}}\right) \\
= & -1 .
\end{aligned}
$$

By Theorem 3.2 of $\mathrm{Wu}$ [14], we conclude that the connected component $l_{\left(z^{*}, \tau_{2 j}^{\prime}, 2 \pi / \omega_{0}\right)}$ through $\left(z^{*}, \tau_{2_{j}}^{\prime}, 2 \pi / \omega_{0}\right)$ in $\Sigma$ is nonempty. Meanwhile, we have

$$
\sum_{(\bar{z}, \tau, p) \in l_{\left(z^{*}, \tau_{2}^{\prime}, 2 \pi / \omega_{0}\right)}} \gamma(\bar{z}, \tau, p)<0
$$

and hence $l_{\left(z^{*}, \tau_{2,}^{\prime}, 2 \pi / \omega_{0}\right)}$ is unbounded.

From (36), we see that, for $j \geq 1,2 \pi / \omega_{0}<\tau_{2_{j}}^{\prime}$. Then, we are in a position to prove that the projection of $l_{\left(z^{*}, \tau^{\prime}{ }_{2 j}, 2 \pi / \omega_{0}\right)}$ onto $\tau$-space is $[\bar{\tau}, \infty)$, where $\bar{\tau}<\tau_{2_{j}}^{\prime}$. Clearly, it follows from the proof of Lemma 9 that system (3) with $\tau=0$ has no nontrivial periodic solution. Hence, the projection of $l_{\left(z^{*}, \tau_{j}^{\prime}, 2 \pi / \omega_{0}\right)}$ onto $\tau$-space is away from zero.

For a contradiction, we suppose that the projection of $l_{\left(z^{*}, \tau_{j}^{\prime}, 2 \pi / \omega_{0}\right)}$ onto $\tau$-space is bounded. This means that the projection of $l_{\left(z^{*}, \tau_{j}^{\prime}, 2 \pi / \omega_{0}\right)}$ onto $\tau$-space is included in an interval $\left(0, \tau^{*}\right)$. Noting that $2 \pi / \omega_{0}<\tau_{2 j}^{\prime}$ and applying Lemma 9, we have $p<\tau^{*}$ for $(z, \tau, p)$ belonging to $l_{\left(z^{*}, \tau_{2 j}^{\prime}, 2 \pi / \omega_{0}\right)}$. This implies that the projection of the connected component $l_{\left(z^{*}, \tau_{2 j}^{\prime}, 2 \pi / \omega_{0}\right)}$ onto $p$-space is bounded. In addition, from Lemma 8 , we obtain that the projection of $l_{\left(z^{*}, \tau_{2}^{\prime}, 2 \pi / \omega_{0}\right)}$ onto $z$-space is bounded if the projection of $l_{\left(z^{*}, \tau_{2}^{\prime}, 2 \pi / \omega_{0}\right)}$ onto $\tau$-space is bounded. Thus, the connected component $l_{\left(z^{*}, \tau_{2 j}^{\prime}, 2 \pi / \omega_{0}\right)}$ crossing through $\left(z^{*}, \tau_{2_{j}}^{\prime}, 2 \pi / \omega_{0}\right)$ is bounded, which is a contradiction. This implies that the projection of $l_{\left(z^{*}, \tau_{2}^{\prime}, 2 \pi / \omega_{0}\right)}$ onto $\tau$-space is $[\bar{\tau}, \infty)$ for each $j \geq 1$, where $\bar{\tau}<\tau_{2_{j}}^{\prime}$. This is the end of the proof.

\section{Conclusions}

In this paper, we investigate the effect of the time delays $\tau_{1}$ and $\tau_{2}$ on the stability of the positive equilibrium of 
system (3) and derive the direction and stability of the Hopf bifurcation. Numerical simulations are carried out to illustrate the theoretical prediction and to explore the complex dynamics including chaos. Finally, we study the global continuation of periodic solutions bifurcating from the point $E_{*}$ for $\tau_{1}$ is fixed in the interval $\left(0, \tau_{1_{0}}\right)$ and show the global existence of the periodic solutions.

\section{Conflict of Interests}

The authors declare that there is no conflict of interests regarding the publication of this paper.

\section{Acknowledgments}

The authors are grateful to the reviewers for their valuable comments and suggestions which have led to an improvement of this paper. This research is supported by the National Natural Science Foundation of China (Grant no. 11061016).

\section{References}

[1] P. H. Leslie, "A stochastic model for studying the proper ties of certain biological systems by numerical methods," Biometrika, vol. 45, no. 1-2, pp. 16-13, 1958.

[2] Y. L. Song, S. L. Yuan, and J. M. Zhang, "Bifurcation analysis in the delayed Leslie-Gower predator-prey system," Applied Mathematical Modelling, vol. 33, no. 11, pp. 4049-4061, 2009.

[3] E. Beretta and Y. Kuang, "Global analyses in some delayed ratiodependent predator-prey systems," Nonlinear Analysis: Theory, Methods and Applications, vol. 32, no. 3, pp. 381-408, 1998.

[4] Q. Wang, M. Fan, and K. Wang, "Dynamics of a class of nonautonomous semi-ratio-dependent predator-prey systems with functional responses," Journal of Mathematical Analysis and Applications, vol. 278, no. 2, pp. 443-471, 2003.

[5] J. M. Cushing, Integro Differential Equations and Delay Models in Population Dynamics, Springer, Heidelberg, Germany, 1977.

[6] S. A. Gourley and M. V. Bartuccelli, "Parameter domains for instability of uniform states in system with many delays," Journal of Mathematical Biology, vol. 35, no. 7, pp. 843-867, 1997.

[7] W. Yu and J. Cao, "Stability and Hopf bifurcation analysis on a four-neuron BAM neural network with time delays," Physics Letters A, vol. 351, no. 1-2, pp. 64-78, 2006.

[8] S. Toaha and M. A. Hassan, "Stability analysis of predatorprey population model with time delay and constant rete of harvesting," Punjab University Journal of Mathematics, vol. 40, pp. 37-48, 2008.

[9] R. Xu and Z. Ma, "Stability and Hopf bifurcation in a predatorprey model with stage structure for the predator," Nonlinear Analysis: Real World Applications, vol. 9, no. 4, pp. 1444-1460, 2008.

[10] S. Yuan and Y. Song, "Stability and Hopf bifurcations in a delayed Leslie-Gower predator-prey system," Journal of Mathematical Analysis and Applications, vol. 355, no. 1, pp. 82-100, 2009.

[11] S. Yuan and Y. Song, "Bifurcation and stability analysis for a delayed Leslie-Gower predator-prey system," IMA Journal of Applied Mathematics, vol. 74, no. 4, pp. 574-603, 2009.
[12] A. F. Nindjin, M. A. Aziz-Alaoui, and M. Cadivel, "Analysis of a predator-prey model with modified Leslie-Gower and Hollingtype II schemes with time delay," Nonlinear Analysis: Real World Applications, vol. 7, no. 5, pp. 1104-1118, 2006.

[13] B. Hassard, N. Kazarinoff, and Y. H. Wan, Theory and Applications of Hopf Bifurcation, Cambridge University Press, Cambridge, UK, 1981.

[14] J. Wu, "Symmetric functional differential equations and neural networks with memory," Transactions of the American Mathematical Society, vol. 350, no. 12, pp. 4799-4838, 1998.

[15] B. Liu and H. J. Marquez, "Razumikhin-type stability theorems for discrete delay systems," Automatica, vol. 43, no. 7, pp. 1219$1225,2007$. 


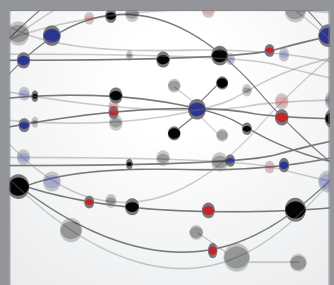

The Scientific World Journal
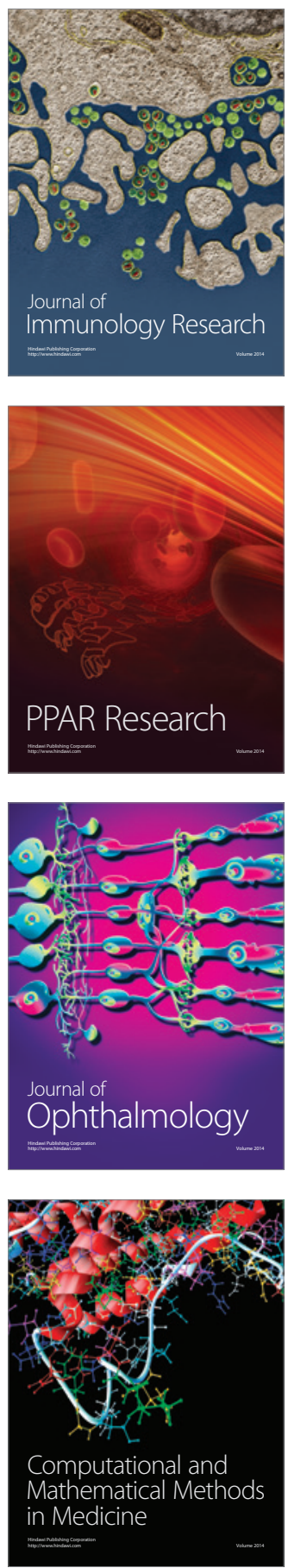

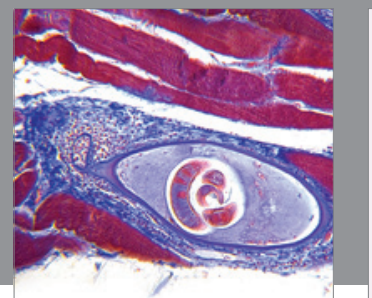

Gastroenterology

Research and Practice
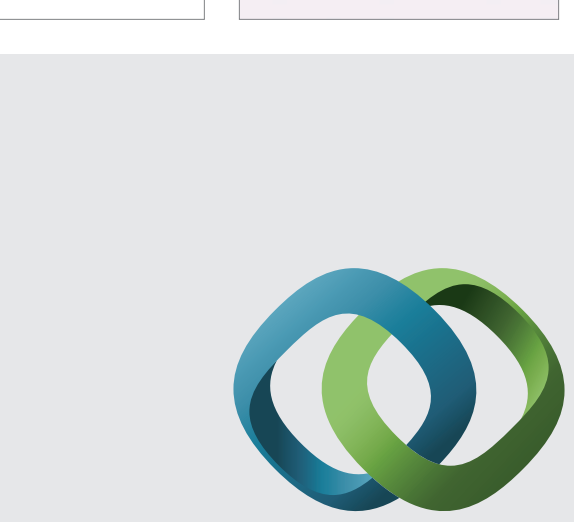

\section{Hindawi}

Submit your manuscripts at

http://www.hindawi.com
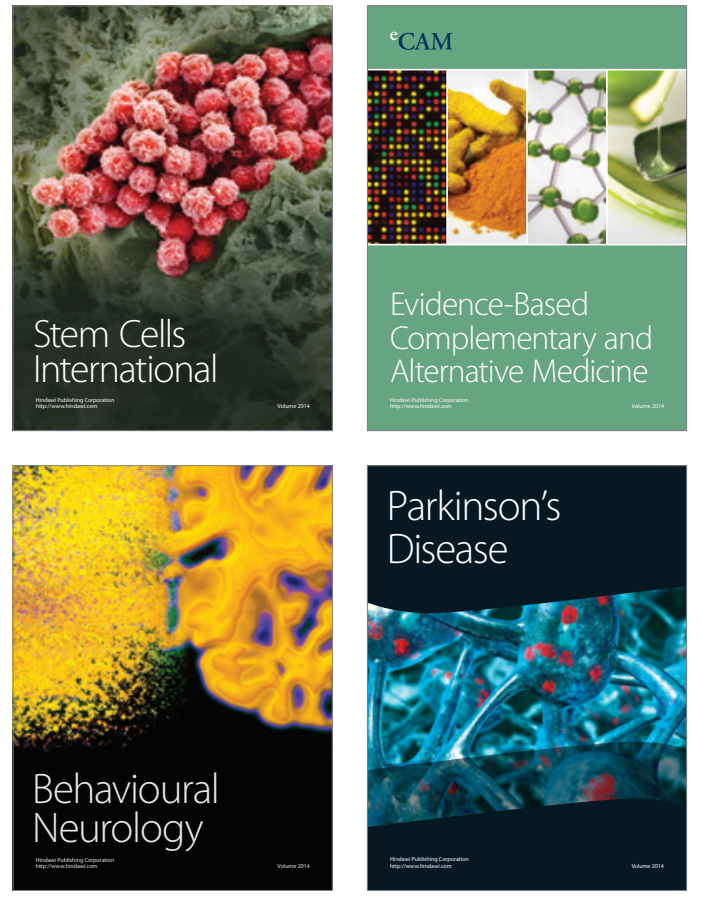
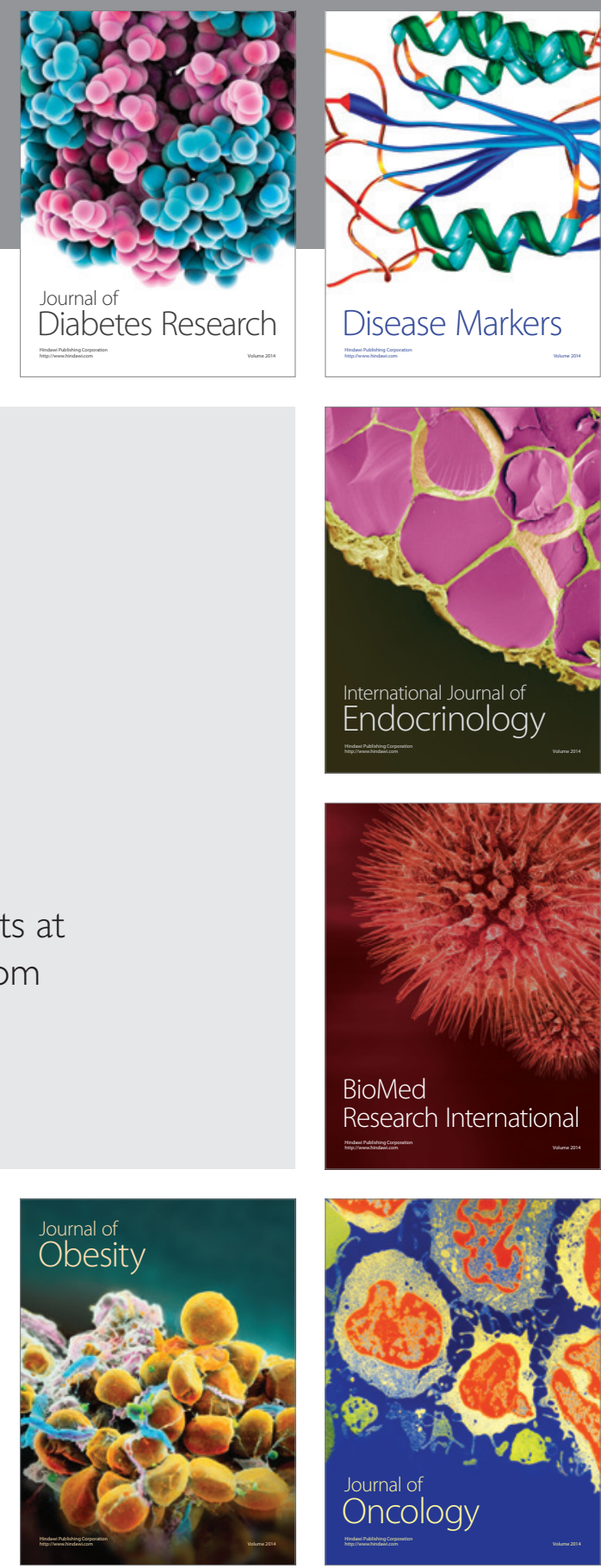

Disease Markers
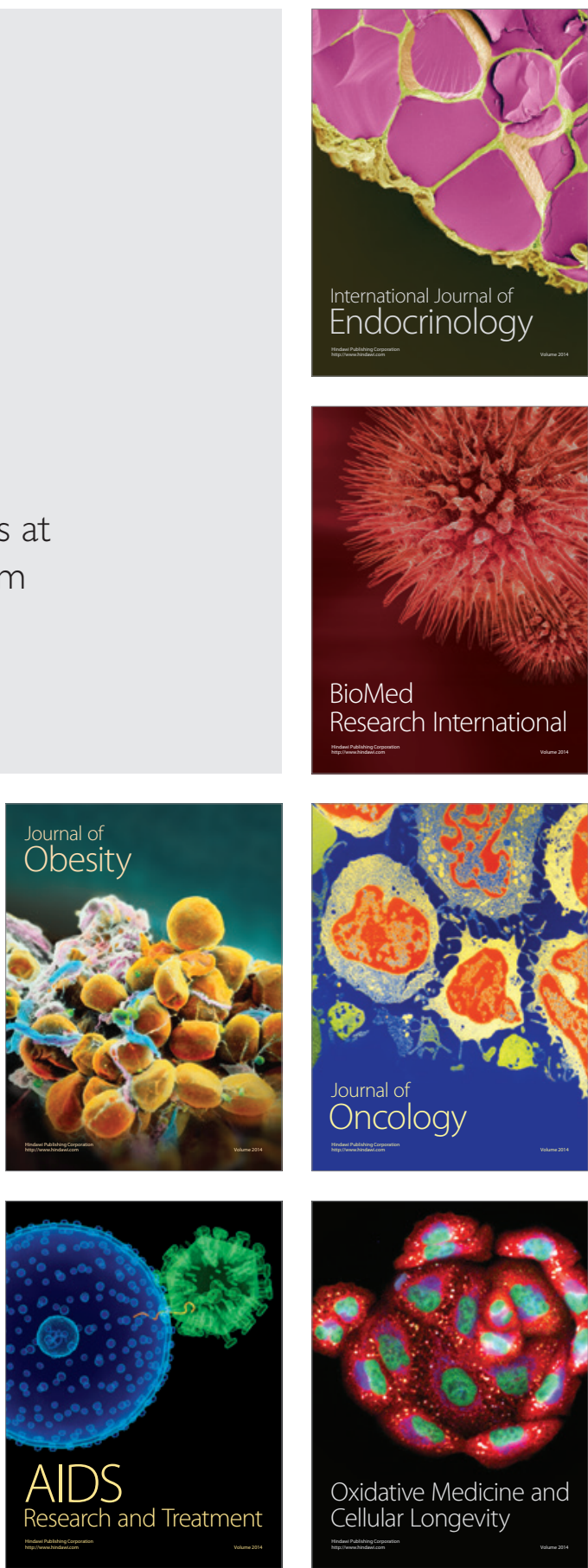